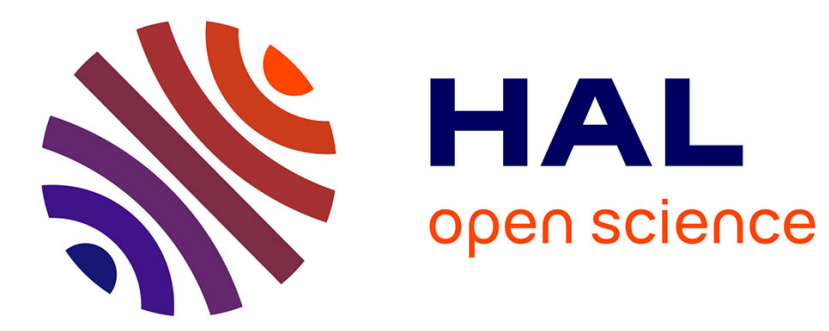

\title{
Méthode de recherche hypothétique et processuelle
}

Celine Racin, Manuella de Luca

\section{To cite this version:}

Celine Racin, Manuella de Luca. Méthode de recherche hypothétique et processuelle. In Analysis, 2020, 4 (1), pp.42-53. 10.1016/j.inan.2019.07.005 . hal-03008733

\section{HAL Id: hal-03008733 https://hal.science/hal-03008733}

Submitted on 16 Nov 2020

HAL is a multi-disciplinary open access archive for the deposit and dissemination of scientific research documents, whether they are published or not. The documents may come from teaching and research institutions in France or abroad, or from public or private research centers.
L'archive ouverte pluridisciplinaire HAL, est destinée au dépôt et à la diffusion de documents scientifiques de niveau recherche, publiés ou non, émanant des établissements d'enseignement et de recherche français ou étrangers, des laboratoires publics ou privés. 


\title{
Méthode de recherche hypothétique et processuelle
}

\section{Hypothetical and processual research method}

\author{
C. Racin ${ }^{\mathrm{a}, *}$, M. De Luca ${ }^{\mathrm{b}}$ \\ ${ }^{a}$ Psychologue clinicienne, hôpital Sainte-Périne-APHP), maître de conférences en psychologie clinique et psychopathologie, faculté de Psychologie, laboratoire \\ subjectivité, lien social et modernité (SuLiSoM-EA3071), université de Strasbourg, 12, rue Goethe, 67000 Strasbourg, France \\ b Psychiatre, responsable du pôle de psychiatrie et de psychopathologie de l'adolescent et du jeune adulte, institut MGEN La Verrière, professeur associé, \\ institut de psychologie, laboratoire psychologie clinique, psychopathologie, psychanalyse (PCPP-EA4056), université Paris-Descartes-Sorbonne Paris-Cité, 71, \\ avenue Édouard-Vaillant, 92100 Boulogne-Billancourt, France
}

I N F O A R T I C L E

Historique de l'article :

Reçu le 11 mars 2019

Accepté le 4 juillet 2019

Disponible sur Internet le 21 août 2019

\section{Mots clés :}

Méthodologie de recherche

Processualité

Hypothèses

Psychanalyse

\section{R É S U M É}

Contexte. - La multiplicité actuelle des méthodes de recherche dans le champ de la clinique référée à la psychanalyse court le risque d'un positionnement qui prône la superposition des modèles en abolissant les antagonismes pour mieux valoriser les complémentarités, donnant parfois l'illusion d'une profusion méthodologique dépliant le champ des possibles.

Objectif. - Cet article se propose de revenir sur les soubassements épistémologiques de ces modèles qui remet invariablement sur le chantier l'adéquation des critères de scientificité retenus, dans le but de se déprendre d'un rabattement syncrétique qui participerait sinon à l'appauvrissement de cette hétérogénéité structurante. Par ailleurs, loin de nous défier a priori des principes méthodologiques issus du modèle expérimental, qui tendent à s'imposer actuellement dans le champ des sciences humaines et sociales, cet article vise néanmoins à se départir du risque hégémonique, ou tout au moins d'une homogénéisation croissante et impérieuse liée à la standardisation de la publication scientifique, en examinant les voies de passage qui permettrait un dialogue fécond aux frontières de la psychanalyse. Méthode. - L'article questionne les soubassements épistémologiques, d'une part, de deux méthodologies régulièrement rencontrées dans le champ clinique - la méthode du cas unique et la méthode hypothético-déductive - puis, d'autre part, de modèles issus historiquement de l'anthropologie et de la sociologie - modèle de la théorisation ancrée et modèle de la traduction élargie - qui partagent avec notre approche le souci de la défense et de la valorisation de la recherche qualitative.

Résultats. - La mise en tension de ces différents modèles est mise au service du dégagement des apports et des limites susceptibles d'éclairer les enjeux épistémologiques spécifiques, les choix d'opérationnalisation méthodologique et les choix d'exposition des résultats des recherches cliniques se référant à la psychanalyse. L'article soutient l'intérêt, pour la psychanalyse, de trouver une opportunité de structuration de ses travaux autour du maintien d'un homomorphisme entre processus psychanalytique et processus de recherche. Ces considérations nous amènent à faire porter 1'attention sur les conditions de possibilité requises pour inscrire la processualité ainsi que l'exigence de maintien d'un écart théoricoclinique au cœur de la méthode de recherche clinique. Dans cette voie, l'article pose les prémisses d'une modélisation d'une méthode de recherche hypothétique et processuelle fondée sur la prise en compte de l'appréhension complexe et nécessairement asymptotique de l'inconscient. Dans cette perspective, le temps de l'écriture de la recherche revêt une double fonction: il redouble, d'une part, les effets de processualité et d'après-coup du dispositif de recherche; il aménage, d'autre part, à travers le choix d'exposition méthodologique des résultats, des conditions communes de débats promptes à situer les différentes partitions du dialogue à plusieurs au sein d'un champ de controverse.

Conclusion. - Cet article propose une première étape de formalisation d'une " méthode de recherche hypothétique et processuelle» susceptible d'offrir une narration qui intègre tout à la fois les contraintes inhérentes à la dynamique de production singulière de la connaissance dans le champ clinique référé à la psychanalyse et celles inhérentes à la possibilité d'un débat scientifique élargi.

\footnotetext{
* Auteur correspondant.

Adresses e-mail : mdeluca@mgen.fr (M. De Luca).
} 
Keywords:

Research method

Processuality

Hypothesis

Psychoanalysis.
The current multiplicity of research methods in the field of clinical psychoanalysis runs the risk of advocating the superposition of models by abolishing antagonisms to better accentuate complementarities, sometimes giving the illusion of a methodological profusion expanding the field of possibilities. Goal. - This article proposes to return to the epistemological foundations of these models, which invariably questions the adequacy of the selected scientific criteria. It aims to get rid of a syncretic drawdown that would otherwise contribute to the impoverishment of this structuring heterogeneity. The methodological principles derived from the experimental model are now becoming established in the field of human and social sciences. Far from challenging this model, this article nevertheless aims to question this hegemonic risk, or at least an increasing and imperative homogenization linked to the standardization of scientific publication, by examining the passageways that would allow a fruitful dialogue at the borders of psychoanalysis.

Method. - The article questions the epistemological foundations, first, of two methodologies regularly encountered in the clinical field - the single case method and the hypothetical-deductive method - and then, of models historically derived from anthropology and sociology - grounded theory model and extended translation model. The latter share with our approach the concern for the defense and enhancement of qualitative research.

Results. - The tension between these different models is used to identify the contributions and limits likely to shed light on the specific epistemological issues, the choices of methodological operationalization, and the choices of exposure of clinical research results related to psychoanalysis. The article encourages psychoanalysts to find opportunities to structure their work around the maintenance of a homomorphism between psychoanalytic and research processes. These considerations lead us to focus attention on the conditions required to inscribe processuality and the maintenance of a theoretical-clinical gap at the heart of the clinical research method. In this way, the paper lays the foundations for a modeling of a hypothetical and process-oriented research method. This method is based on taking the complex and necessarily asymptotic apprehension of the unconscious into account. From this perspective, the writing phase has a dual function: on the one hand, it doubles the effects of processuality and of the "après-coup" of research; on the other hand, the choice of methodological exposure of the results must propose common conditions for debate within a controversial field. Conclusion. - This article proposes a first step in the formalization of a "hypothetical and processual research method." This method is likely to offer a narrative that integrates both the constraints related to the dynamics of the singular production of knowledge in psychoanalysis's clinical field and the constraints promoting a broader scientific debate.
Il m'importe peu que la psychanalyse ne soit pas admise dans le cercle des sciences «dures » ou «molles », ni même qu'elle soit dénoncée ici et là comme une «fausse science », pourvu qu'elle reste inventive et jeune, telle Gradiva rediviva, au pas vif et décidé !

(Pontalis, 2012, p. 180).

\section{Introduction}

Si la place de la psychanalyse au sein des sciences humaines est sans cesse remise en cause et notamment parmi certains enseignants-chercheurs universitaires, la reconnaissance et la mise en avant de ses spécificités comme mode de pensée et d'accès à la connaissance nous semble un enjeu non négligeable pour élaborer des recherches référées à la psychanalyse. Les modalités des rencontres réalisées en recherche clinique, structurellement dépendantes de leurs finalités, exigent une explicitation du positionnement épistémologique, précédant rigoureusement le positionnement méthodologique qui permet de répondre à l'interrogation subséquente : quelle est la relation du chercheur avec l'objet de recherche ? Cette réflexion importante se trouve, paradoxalement, très souvent occultée dans la démarche qui vise à envisager, pour le chercheur, un certain rapport méthodologique à la réalité, c'est-à-dire de déterminer par quel moyen accéder à la connaissance de l'objet, par quelle instrumentation rendre compte de la relation avec son objet d'étude. Force est de constater aujourd'hui l'émergence grandissante d'« arrangements méthodologiques » multiples empruntant à divers modèles et conduisant à des positions que l'on pourrait qualifier d' " aménagées ${ }^{1}$ (à défaut d'être réconciliables). Cet aménagement des paradigmes, hautement discutable de notre point de vue, rend d'autant plus nécessaire une réflexion spécifique mettant en évidence les présupposés épistémologiques des recherches cliniques référées à la psychanalyse, dont les implications en termes d'orchestration méthodologique et de statut des résultats ne doivent pas être sous-estimées.

En contrepoint du modèle originel du cas unique comme mode privilégié de production des connaissances dans le champ clinique, modèle dont la scientificité est régulièrement remise en question, d'autres méthodes s'offrent actuellement comme alternatives en donnant parfois l'illusion d'une profusion méthodologique dépliant le champ des possibles. Si le dépassement de certaines dichotomies est de plus en plus appelé, questionnant par exemple les rapports du particulier au général, de l'objectif au subjectif, etc. à travers une hybridation des raisonnements (Perron, 2010 ; Poenaru, 2018), nous souhaitons de notre côté revenir sur les postulats épistémologiques sous-jacents aux modèles les plus discutés aujourd'hui dans le champ clinique, la méthode expérimentale et le modèle de la théorisation ancrée, en mettant en exergue les limites qui fondent selon nous leur incompatibilité avec la référence à la psychanalyse. Le cadre de cet article ne

\footnotetext{
${ }^{1}$ L'article de Philippe Baumard suggère ainsi qu'en sciences de gestion, " le constructivisme à la française est avant tout un "positivisme aménagé" et, d'autre part, qu'il n'y a pas engagement ou positionnement épistémologique ex ante, mais glissement progressif d'un épistèmê positif à une praxis constructiviste » (1997, p. 2).
} 
permettant pas un exposé exhaustif des controverses scientifiques entourant le champ de la clinique référée à la psychanalyse, nous discuterons particulièrement ces modèles scientifiques en vue de situer notre proposition d'une " méthode de recherche hypothétique et processuelle». Ainsi, dans le modèle expérimental qui tend à s'imposer comme référence scientifique, nous soulignons notamment son postulat selon lequel la réalité est considérée comme objective, composée de faits stables dans 1'absolu. Sur un pô le constructiviste opposé à ce pôle réaliste, la théorisation ancrée, dans son acception initiale et standard $^{2}$, renvoie à un courant herméneutique reposant sur un rapport subjectif, construit, à la réalité, et donc sur une réalité instable, dépendante des sujets qui lui donnent son sens. «Le rapport au sens, dans la recherche qualitative [référée à la théorisation ancrée], renvoie au courant herméneutique et plus précisément à l'idée d'une “double herméneutique', dans la mesure où il s'agit pour le chercheur d'interpréter des faits auxquels les sujets observés ont déjà donné du sens. Mucchielli (2004) parle d'un “construit de second degré", à savoir d'une construction scientifique à partir "d'un fait signifiant déjà construit par une collectivité humaine"” " (Pelaccia \& Paillé, 2009), un fait entendu ici dans le sens de «ce qu'on a fabriqué » (Latour, 2009).

Or, dans notre perspective et en nous inspirant des travaux de B. Latour, le choix dans la recherche clinique ne se situe pas «entre réalisme et constructivisme, il est entre ce choix lui-même et l'existence pratique qui n'en comprend ni l'énoncé ni l'importance » (ibid.). Nous retrouvons ici l'écart dénoncé par l'auteur, notamment à partir de son étude du travail scientifique de L. Pasteur, «somm[é] de choisir entre constructivisme et réalisme. Ou bien il a construit socialement ses faits de toutes pièces et il n'ajoute donc au répertoire du monde aucune autre réalité que celle de ses fantasmes, préjugés, habitudes et mémoire; ou bien les faits sont réels, mais alors il ne les a pas fabriqués de toutes pièces dans son laboratoire [en les mettant en scène dans les dispositifs ad hoc qu'il a construits] » (ibid.). Le modèle de la traduction élargie, sur lequel nous nous attardons ensuite, nous semble particulièrement intéressant à convoquer : loin d'amalgamer les deux pô les antagonistes que sont le constructivisme et le réalisme, ou de les abouter à travers un «feuilletage des intermédiaires » (Latour, 1991), la réflexivité sur la recherche s'appuie sur un engagement ontologique particulier selon lequel la réalité repose sur des chaînes de traduction et de médiation. Dans ce modèle, le chercheur met en place des dispositifs dont la finalité est de suivre processuellement ces chaînes de traduction, et donc ces interactions dynamiques, en appréhendant leurs modalités de stabilisation et de déstabilisation, saisies dans un même mouvement et non pas de façon fractionnée, clivée, puis recomposée a posteriori.

Cet article vise donc à exposer ces différents points de contextualisation pour avancer ensuite notre proposition d'une « méthode de recherche hypothétique et processuelle».

Prolégomènes : des méthodologies de recherche utilisées dans le champ de la clinique

Les méthodologies de recherche utilisées dans le champ de la clinique sont multiples. Deux d'entre elles ont retenu notre attention en raison de leur prédominance dans les recherches menées actuellement.

${ }^{2}$ Il existe également une acception différente de la théorisation ancrée, dans laquelle la réalité reste stable et est appréhendée méthodologiquement par induction. Les positionnements ontologiques et épistémologiques restent proches de la perspective hypothético-déductive et se situent, dans tous les cas, à l'intérieur du positivisme scientifique.
Aux commencements : le cas unique

Historiquement, la méthode du cas unique a été dominante dans les différents champs clinique, médical, psychiatrique, psychologique ou psychanalytique. Après avoir été la cible de critiques méthodologiques concernant son manque d'objectivité et de scientificité, elle fait l'objet depuis peu d'un regain d'intérêt dans la recherche en sciences sociales (Passeron \& Revel, 2005). Pour en souligner la spécificité, D. Widlöcher (1990) propose d'utiliser le terme de «cas singulier», mettant ainsi l'accent sur la dimension individuelle, unique de la description, son caractère remarquable, c'est-à-dire hors du commun, emblématique, paradigmatique, complexe voire à l'origine de butées ou d'achoppements face aux données classiques. Cette méthodologie n'est cependant pas l'apanage des disciplines cliniques, elle est aussi utilisée dans le champ des sciences sociales comme en histoire, en sociologie, mais également des mathématiques ou du droit.

La méthode des cas cliniques ouvre sur une interrogation concernant la généralisation et la dynamique permettant le passage et 1'extension de configurations singulières à celles plus universelles. Pour Passeron et Revel (2005), « le penser par cas » ouvre à une voie tierce de connaissance, à « une figure intermédiaire de rationalité ». Ce que nous avons proposé comme recherche d'invariants dans des recherches portant l'une sur le recours aux scarifications (De Luca, 2009), et 1'autre sur le vécu de la dépendance dans le grand vieillissement (Racin, 2017), nous semble relever de cette voie intermédiaire qui procède par études de multiples cas pour en repérer les singularités, les différences, les similitudes et les répétitions dans le cours du fonctionnement psychique. Cette recherche d'invariants s'élabore dans le croisement des coordonnées métapsychologiques - économiques, topiques, dynamiques - et de l'histoire de vie de chacun. Le travail, proposé par J.-M. Thurin (1996), de formalisation du recueil de données dans un cadre de psychothérapie et de psychanalyse, s'inscrit dans ce courant. La prise en compte dynamique du déroulement de la thérapie et des enjeux intersubjectifs complète le recueil habituel de l'histoire du patient, du récit de ses rêves et de certains verbatim de séances. Ainsi, dans cette méthodologie, il s'agit pour J.-M. Thurin non pas de viser " "la preuve", ; mais des observations convergentes sur un objet commun peuvent avoir un effet de connaissance assorti de conséquences pratiques. Il s'agira alors de définir le plus précisément possible ce que l'on étudie et comment» (op. cit., p. 2). La méthode du cas unique a de nouveau sa place mais la mise en place d'hypothèses dans cette méthode de recherche - nous y reviendrons - est aussi soulignée par 1'auteur.

Ce passage du singulier à l'universel ne se fait pas uniquement par extension à un groupe ou une cohorte de sujets; il procède aussi de ce qu'A. Green décrit de la pensée clinique, de son fonctionnement par associativité et de son pouvoir d'évocation auprès d'autres cliniciens : " on reconnaît indubitablement la pensée clinique quand l'élaboration théorique soulève des associations qui se réfèrent à tel ou tel aspect de l'expérience psychanalytique chez le lecteur » (2002, p. 11). L'extension se fait par contiguïté, par alliance, par assemblage, par combinaison des invariants, sans pour autant opérer une réduction induite par une volonté de généralisation à tout prix. En effet, celle-ci entraîne une homogénéisation du matériel et des résultats, faisant courir le risque de simplification, d'amputation ou d'abrasion des éléments originaux et remarquables du cas. L'ouverture sur le général ne se fait pas sur l'intégralité des données recueillies mais sur certaines d'entre elles - les invariants - alors mises en avant car virtuellement présentes chez d'autres sujets ou s'y déployant de manière similaire.

Les cas uniques, historiquement sollicités comme méthodes au plus près des avancées théoriques freudiennes, font maintenant l'objet d'une désaffection et de nombreuses critiques. Cette 
méthode serait désuète, témoin d'une période révolue où les pionniers de la psychiatrie et de la psychanalyse défrichaient le champ de la clinique en accumulant des cas cliniques, faute de véritables recherches épidémiologiques. Lui sont reprochés pê lemêle son manque de reproductibilité, la mise en avant de son particularisme, son recours à un échantillon non représentatif. Une certaine scientificité se réclame d'études de cohorte, d'analyses statistiques avec le nombre comme garantie au risque de réductionnisme comme scotome. Cette perspective promeut une vision de la moyenne et de l'homogénéité au détriment du singulier et des bigarrures de la psyché. Cependant, la méthode du cas construit peut revêtir tout à la fois une valeur d'exemple, d'exception et s'inscrire dans une série. Cette mise en tension entre cas singulier et cas multiples ne s'oppose pas mais s'inscrit dans un paradoxe puisque comme le propose C. Cyssau (1999), «l'observation clinique de nombreux patients, selon la technique analytique, conduira à la construction de l'événement singulier et fortuit du cas. Venant déclasser la théorie préexistante, celui-ci va générer par son dérangement, la découverte [...] qui propulse une nouvelle hypothèse conceptuelle ». Ce qui est ainsi promu et recherché n'est pas la validité par le nombre et la généralisation des données recueillies, mais la prise en compte au sein de nombreux cas de nouvelles propositions théoriques.

Le modèle hypothético-déductif : le modèle expérimental comme référence scientifique

Le modèle hypothético-déductif répond en partie aux critiques $\mathrm{du}$ cas unique mais en attire d'autres en raison d'une trop grande proximité avec un modèle médical causaliste. Ces critiques se condensent sur la mise en place d'hypothèses jugées trop artificielles car pensées en amont des rencontres sur le terrain clinique. La principale porte donc sur la part déductive de la méthode, même si la formulation et la formalisation d'hypothèses ne fait pas l'unanimité dans le champ de la clinique en raison de leur part trop rigide et trop artificielle.

Le paradigme hypothético-déductif s'inscrit dans une vision rationaliste et scientifique de la connaissance. Il s'oppose à l'empirisme et à sa méthodologie inductive : les observations concrètes permettent de produire une théorie générale abstraite qui est «vérifiée » par de nouvelles observations. Les hypothèses portent sur cette réalité concrète et supposée objective : elles sont construites à partir de pré-conceptions (basées ou non sur des expérimentations); elles sont formulées sous la forme de probabilités censées être vérifiables - la notion de vérifiabilité renvoyant à plusieurs types de conceptions selon les auteurs, dont celle de réfutabilité (Borst \& Cachia, 2018 ; Kalat, 1999; Popper, 1934) - par le protocole expérimental. Les hypothèses, une fois supposées vérifiées, sont présumées révéler une part de la vérité sur un monde objectivable. La déduction apparaît quant à elle, dans la méthode hypothético-déductive, un mode de production des résultats dans une reproduction du traitement des données de l'expérimentation. Elle ne peut cependant se concevoir sans référence à une théorie sous-jacente qui permet qu'elle puisse advenir sans se réduire à des spéculations, point de vue souligné par Bachelard (1934), pour qui il n'y a pas d'expérience sans théorie sous-jacente support de son déchiffrage. Pour Freud, dans l'Abrégé, " toute science repose sur des observations et des expériences que nous transmet notre appareil psychique, mais comme c'est justement cet appareil que nous étudions, l'analogie cesse ici. Nos observations se pratiquent à l'aide du même appareil de perception et nous nous servons précisément des lacunes dans les séries des processus psychiques. Nous reconstituons, en effet, ce qui manque, par des déductions plausibles et le traduisons en matériel conscient. [...] La certitude relative de notre science du psychisme repose sur la puissance convaincante de ces déductions »(1938, p. 21-22). Si Freud ne réfute pas l'utilisation de l'expérimentation et de la déduction, il souligne les limites de l'analogie dans la nécessaire prise en compte de l'implication du chercheur et de son contre-transfert face au matériel observé. Il met également en exergue la butée représentée par l'insaisissable de l'inconscient, tout comme la tentation d'un rabattement dont est porteuse l'analogie avec la méthode expérimentale et le recours prévalent à la déduction. Le chercheur expérimentateur, doit veiller, dans la méthodologie hypothético-déductive, à ne pas perturber le protocole expérimental mis en place, sa subjectivité n'étant pas engagée dans la production et l'analyse des résultats.

Le fonctionnement psychique se caractérise par sa complexité en raison notamment de l'intrication permanente entre phénomènes conscients et inconscients. Comment faire face à cette complexité sans risquer la simplification en raison d'une tendance à la réification des phénomènes ou au recours à l'analogie ? Comment faire sien le paradoxe de 1'hyper-complexité avancé par H. Atlan qui invite à " aller, non pas du simple au complexe, mais de la complexité vers toujours plus de complexité » (1979, p. 191-194) ? Le maintien et la mise au travail d'un écart entre les théorisations proposées - quelle que soit leur origine : théorie du chercheur, théorie référencée à une école - et la pratique de la recherche clinique, représente un début de réponse à cette complexité. Chaque recherche tente de réduire cet écart par les propositions qu'elle formule tout en sachant que la présence de cet écart soutien l'intérêt de la recherche. La connaissance de l'inconscient et des processus psychiques reste asymptotique et ne peut être totalisante, mais incite le chercheur à penser des dispositifs de recherche et des démarches méthodologiques variés. Des travaux conjoints de psychanalystes et de neuroscientifiques (Ansermet \& Magistretti, 2004) offrent la possibilité de penser cet écart et cette complexité en gardant la spécificité de chaque épistémologie et en la dialectisant au service d'une fécondité de la pluralité des points de vue. Ces modalités de recherche transdisciplinaires ne peuvent résumer les recherches cliniques en psychanalyse et l'écart peut et doit se construire à l'intérieur même d'un référentiel analytique.

La méthode hypothético-déductive met à l'épreuve la validité des hypothèses par la mise en œuvre d'un protocole expérimental/ de recherche. Leur non-validation rend caduque les hypothèses, ce qui fragilise la méthode en raison de l'absence de prise en compte de l'inévitable écart théorico-clinique ou pratique; elle tend à inciter à formuler les hypothèses le plus tardivement possible. La méthode hypothético-déductive peut donc être utilisée dans des recherches cliniques adossées à la psychanalyse à la condition que puisse se maintenir un écart entre la formulation des hypothèses et la technique utilisée pour les analyser. La prise en compte de la part de subjectivité du chercheur et la nécessaire formulation des hypothèses au début de la recherche, sans que celle-ci ne soient transformées en cours de recherche - en raison d'une noncongruence de la clinique ou de la découverte d'un nouvel éclairage théorique - en sont quelques-uns des garants.

L'utilisation de la déduction reste le point faible de cette méthode puisque la confrontation à la recherche clinique ne peut être équivalente à un protocole expérimental où les résultats sont déduits par observation. L'observation en clinique est ultrascopique, menée indissociablement de l'écoute. Elle prend en compte le manifeste et le latent, le verbal et le non-verbal et tente ainsi de repérer les traces des processus inconscients. Le recours à une double investigation - par des entretiens de recherche et des tests projectifs par exemple - participe à cet objectif d'une analyse au-delà de la simple observation visuelle et permet une «plongée dans les profondeurs » de la psyché, même si cette modalité de recherche ne peut se généraliser à l'ensemble des recherches ou des chercheurs en clinique. 
Enjeux épistémologiques : quelques modèles aux frontières de la psychanalyse

L'acte même d'écouter parle, écrit, imprime sur les archives la marque d'un événement, surajouté aux traces dont l'écoute se voudrait seulement la captation (D. Scarfone, 2004a, p. 111).

\section{Le modèle de la théorisation ancrée}

La théorisation ancrée connaît depuis une dizaine d'années un engouement dans les recherches en science sociales (Glaser \& Strauss, 1967) et plus particulièrement en psychologie clinique, même si sa duplication dans le champ des recherches en clinique limite voire gomme leur spécificité que représente la prise en compte des effets de la rencontre intersubjective. Issue de la sociologie et des recherches dans le milieu médical, elle offre une alternative séduisante à la méthodologie hypothético-déductive. Elle se définit comme "visant à générer inductivement une théorisation au sujet d'un phénomène culturel, social ou psychologique, en procédant à la conceptualisation et la mise en relation progressives et valides de données empiriques qualitatives " (Paillé, 1994). Il s'agit donc d'une méthode qualitative inductive dans laquelle, contrairement aux méthodes hypothético-déductives, il n'y a pas d'hypothèse à vérifier mais un enrichissement progressif de la connaissance d'un phénomène par la confrontation aux données du terrain. Le point de départ est un intérêt du chercheur autour d'un questionnement. Le sujet précis de la recherche ne peut être déterminé au préalable. Seul un champ de recherche est prédéfini. Celui-ci est adossé à la clinique et nécessite une suspension des acquis et des connaissances. Dans un premier temps, la théorie est laissée de côté pour permettre une imprégnation totale du chercheur par les observations recueillies sur le terrain. Le passage du singulier au général se fait par inférence inductive et implique le recueil d'un grand nombre de données congruentes aux premières. Il s'agit donc de réaliser un nombre important d'observations, réalisation qui se déroule dans des conditions variées, sans qu'aucune de ces observations ne vienne contredire les autres - ce qui constitue à notre sens une des faiblesses de cette méthodologie, nous y reviendrons.

Les résultats sont enracinés dans les données, ils se constituent dans des allers et retours permanents entre terrain et avancée de la recherche, dans une comparaison entre la collecte et l'analyse des données. Une première formulation provisoire est proposée pour comprendre la complexité des phénomènes, tant au niveau conceptuel qu'au niveau empirique de ces mises en situation. Contrairement à ce qui se pratique d'ordinaire, l'analyse par théorisation ancrée ne débute pas après l'étape du recueil de données, mais en même temps que la constitution de catégories. Ces catégories sont constamment soumises à l'avancement dans le recueil de données et peuvent évoluer autant que nécessaire.

Au-delà de l'effet de mode et de séduction d'une méthode de recherche dans laquelle ni référence théorique initiale ni hypothèse ne sont nécessaires, cette méthode permet une appréhension plus sensible de la complexité des phénomènes observés dans la pratique clinique. Elle peut aussi avoir un effet de réassurance sur le chercheur qui ne doit pas exposer ses propres théories avant de les confronter à la réalité du terrain et à sa complexité. Elle offre une garantie de validité, dans un présupposé théorique d'un terrain porteur de vérité, terrain ne pouvant se tromper ou tromper en raison de sa place centrale dans les phénomènes étudiés. On peut cependant, en suivant le paradoxe de Quine (1977), souligner que des observations multiples réalisées à partir de théories différentes, opposées voire contradictoires, valident l'ensemble des théories. Le terrain et son observation n'ont donc pas de valeur de preuve intrinsèque.

Parmi les critiques adressées à la méthodologie par théorisation ancrée, celles portant sur le codage et la constitution de catégories sont nombreuses (Ayache \& Dumez, 2011). Le codage est une démarche très subjective - plusieurs expériences de double codage le démontrent —, de même que la constitution de catégories. Pour pallier cet inconvénient, les chercheurs peuvent constituer des groupes de chercheurs pour réaliser un codage multiple et ainsi définir des catégories moins aléatoires, mais l'appartenance à un groupe de chercheurs mobilisés renvoie toujours à l'existence d'un partage théorique et d'un référentiel commun, ce qui ne limite donc que partiellement le biais induit par la subjectivité du codage. D'autre part, il n'existe pas de codage sans référence à une théorie sous-jacente, puisqu'un codage ne se résume pas à l'isolation d'un mot ou d'une partie de matériau : il est un choix du chercheur en référence à une théorie. L'observation par immersion sur le terrain renvoie à des méthodologies utilisées en sociologie mais aussi à une approche clinique au lit du malade, autant de références théoriques incluses d'emblée dans le protocole de recherche et dans le recueil des données, ce qui vient mettre à mal cet idéal de suspension de toute référence théorique. Le chercheur se devrait, comme le psychanalyste décrit par Bion, être "sans désir, sans mémoire, sans compréhension " (1970). Toutefois, cette disposition est à attendre, pour Bion, comme la mise en avant de l'intuition dans le processus analytique. À cet endroit, les modes de recours au codage et à la catégorisation défendus par le modèle de la théorisation ancrée semblent très éloignés d'une intuition comme mode de compréhension et d'appréhension du sujet. Ce refus d'une confrontation initiale à une théorisation, de même que le nom donné à cette méthodologie, posent d'emblée comme but de la recherche la production d'une nouvelle connaissance théorique. Ce but peut être d'éclairer différemment une théorie, mais plus souvent il s'agit de vouloir produire une nouvelle théorie affranchie du carcan de références aux théories existantes.

Une autre critique que nous pouvons formuler porte sur le risque de validation tautologique d'hypothèses construites après l'observation de terrain, ce qui a été observé devenant le résultat de l'hypothèse issue de l'observation, dans une circularité peu propice à l'inattendu, à l'écart, à la différence et aux effets de l'après-coup. Ces effets, comme ceux du retour du refoulé tels que nous pouvons les observer chez des sujets en thérapie, ne peuvent s'arrêter au seuil du psychisme du clinicien devenu chercheur. Ils participent à enrichir les recherches en clinique adossées à la psychanalyse et ne peuvent être abolis car décrétés par la méthodologie. Cette recherche d'une virginité du chercheur peut renvoyer à une volonté de pureté et de non-corruption, pétrie d'un idéal excluant toute référence au sexuel et à sa contribution à la production de théories dès le début de la vie, contribution infiltrant aussi le travail du chercheur.

Enfin, l'observation d'un phénomène, y compris par expérimentation dans des sciences dures comme la physique, entraîne une variation de ce phénomène. La prise en compte du contretransfert du chercheur sur la recherche et sur les sujets de sa recherche permet d'avoir un accès, certes partiel, à ces phénomènes, mais néanmoins indispensable pour les prendre en compte dans le traitement des données et des résultats, là où les opérations de codage et de catégorisation se voudraient suffisamment neutres, au maximum "chercheur indépendant ", pour être reproductibles et donc génératrices de théorisation. La connaissance des phénomènes psychiques, dont une grande partie est inconsciente, doit à un moment intégrer les modalités qui permettent d'y avoir accès. Dans cette perspective, la prise en compte du contre-transfert du chercheur et du transfert des sujets sur le chercheur et sur la recherche participe grandement à ces 
modalités à considérer, ce qui n'est pas intégré dans l'utilisation du codage et de la catégorisation, et commence seulement à l'être lors de la troisième étape, celle de la mise en relation des données. La subjectivité du chercheur y est amplement mobilisée et peut être analysée. Quant à la subjectivité des sujets, celle-ci peut sans doute être intégrée dès l'étape de codage et de constitution des catégories, limitant alors le risque de centrage trop exclusif sur les données manifestes du matériel.

La traduction au cœur du «modèle de la traduction élargie » et du processus psychanalytique

Avant de revenir du cô té de la psychanalyse, nous souhaitons ici attirer l'attention sur les travaux de M. Callon (1995) étonnamment méconnus dans le champ des recherches adossées à la psychanalyse - auquel revient le mérite d'avoir proposé une classification originale des modèles de caractérisation de la science, échappant aux dualismes réductionnistes entre réalisme et constructivisme, entre connaissance objective et connaissance subjective, entre théorie et pratique, dualismes contenus dans l'opposition courante entre méthode hypothético-déductive et méthode par théorisation ancrée. Ces modèles réunissent des auteurs qui contribuent à la philosophie des sciences et aux (social) studies of science (SSS), principalement à partir de travaux en sociologie, en anthropologie et en économie. Au sein de ces modèles, le rapprochement des travaux se justifie du fait des réponses, cohérentes entre elles, qu'ils apportent à six questions ${ }^{3}$ considérées comme centrales par l'auteur dans l'effort de caractérisation qu'ils poursuivent. Nous porterons plus particulièrement ici l'accent sur la manière d'envisager les associations entre les énoncés observationnels et les énoncés théoriques produits par l'activité scientifique, préoccupation qui nous semble au cœur de débats concernant la scientificité de la psychanalyse. La tentation du rapprochement avec le modèle hypothético-déductif (plus sur le plan méthodologique, de surcroît, que véritablement épistémologique) se trouve fortement suggéré, comme nous l'avons précisé, par l'exigence de "rationalité » que certains paradigmes scientifiques élèvent en critère de scientificité nodal. Dans ce cadre, la réflexion du chercheur se porte de manière privilégiée sur la validité des énoncés intermédiaires, produits par la psychanalyse, qui mettent en relation les données de l'observation et les systèmes abstraits mobilisés pour expliquer leur régularité. Ce positionnement a pour conséquence d'aimanter le débat scientifique sur ce qui est représenté par ces énoncés et sur leur validité, évaluée notamment à l'aune de critères de falsifiabilité, de puissance de prédiction, etc. Dans une perspective extrême, ce positionnement considère que ces énoncés existent et se structurent indépendamment de ceux qui les créent. Nous retrouvons ici la thèse de la «connaissance objective » proposée par K. Popper (1972), dans la continuité de l'universalité du cogito cartésien ou des catégories transcendantales kantiennes, cette fameuse «connaissance sans sujet connaissant » évoluant dans un «troisième monde » qui s'affranchit de l'homme et échappe aux sujets mêmes qui la produisent. Le scientifique, dans cette voie, est ainsi envisagé comme un intermédiaire, un simple rouage chargé

\footnotetext{
${ }^{3}$ Les six «questions inévitables» identifiées par M. Callon (1995, p. 202) : Quels sont les produits caractéristiques de l'activité scientifique?

Quels sont les acteurs qui contribuent à cette production? De quelles compétences doivent-ils être dotés pour mener à bien cette activité ?

Comment expliquer le caractère ininterrompu et éventuellement cumulatif de l'activité scientifique?

Comment l'accord sur les connaissances produites est-il obtenu ?

Quelle est la forme d'organisation requise par l'activité scientifique ? (il peut s'agir de l'organisation interne aussi bien que de l'organisation des rapports entre science et société).̇̀ quelle dynamique d'ensemble, à la fois sociale, politique, culturelle et économique, contribue le développement scientifique?
}

de transmettre des énoncés qui établissent des liens avec la réalité dont il rend compte, antérieure à l'observation, sa fonction étant sous-tendue par l'idée que les objets étudiés peuvent parler d'euxmêmes. À l'instar de l'invention de la perspective à la Renaissance, cette conduite prétend reconstruire «logiquement les invariances internes à travers toutes les transformations produites par les déplacements dans l'espace [sur lesquels] reposent toutes les grandes généralisations scientifiques ou lois naturelles » (Ivins, 1985, p. 35), sans que ces déplacements n'impliquent de déformation : la représentation se substitue à la "chose » représentée, la carte devient le territoire, et se fixent sous forme de mobiles immuables aisément manipulables et maîtrisables. Ces derniers finissent par être capitalisés dans une «boîte noire » dont on ne se soucie plus de la complexité interne, pour être retravaillés comme les représentants fidèles des entités dont ils parlent, sans prendre davantage en compte le circuit référentiel long et complexe dont ils constituent seulement le dernier maillon, réseau qui se trouve dès lors invisibilisé, rendu anecdotique. Or, cette activité de traduction, visant à rapporter un énoncé à un autre, à référer une catégorie à une autre, résulte « d'investigations, d'interprétations, d'interactions, de choix qui ne peuvent être abstraits des circonstances particulières dans lesquelles ils prennent place et qui interdisent toute vision étroitement déterministe " (Callon, op. cit., p. 239), ce qui pose l'exigence de l'étude du processus même de leur production dans un réseau complexe et continu de «traductions-représentations » (ibid., p. 241). De ce fait, la sensibilité épistémologique à laquelle sont adossées nombre de recherches référées à la psychanalyse nous semble pouvoir soutenir un dialogue extrêmement fécond avec le «modèle de la traduction élargie»-qui s'inscrit dans la lignée des travaux en "sociologie de la traduction»défendus par Madeleine Akrich, M. Callon et B. Latour - selon lequel :

On peut par certains aspects assimiler la science à une vaste entreprise d'écritures, ou plutôt à un dispositif conçu pour faire écrire, par inscriptions interposées, des entités qu'il devient alors possible de qualifier, de décrire et sur lesquelles ou avec lesquelles on peut envisager d'agir. [...] Les dispositifs à mettre en place varient selon les entités à traduire : l'expérience montre et apprend qu'un quark, un corps souffrant, un gène défaillant, un groupe social humilié ou une strate géologique et ses fossiles n'acceptent d'écrire que s'ils sont pris dans des arrangements qui leur sont adaptés (ibid., p. 237).

Une des vertus de cette proposition, selon B. Latour, est d'envisager sous un nouveau jour la nature du résultat scientifique :

Dire d'un résultat qu'il est «scientifique », c'est nous mener vers un laboratoire (au sens large) où l'on tombe sur un ensemble de témoins rassemblés autour d'un instrument qui permet de recueillir le témoignage d'un autre ensemble d'entités soumises à des épreuves grâce auxquelles elles vont pouvoir participer d'une façon ou d'une autre à ce qu'on dit d'elles (Latour, 2010, p. 189).

Ce qui intéresse par exemple B. Latour, dans le sens ainsi donné au résultat scientifique, c'est qu'il n'attire pas l'attention sur des qualités objectives et subjectives des objets d'étude (cf. le statut ontologique des objets d'étude respectivement considérés par le modèle hypothético-déductif et par le modèle par théorisation ancrée), mais

vers un ensemble de montages qui peuvent tous rater : les témoins peuvent être ambigus, les instruments mal réglés, les épreuves mal choisies, les résultats mal interprétés. Dans cette nouvelle acception de l'adjectif « scientifique », il n'y a plus rien d'automatique dans 
l'extension indéfinie des preuves. Dire d'un montage qu'il est scientifique, c'est lancer la discussion, ce n'est plus, comme avec les autres sens, y mettre fin ; c'est commencer le travail d'extension des faits, ce n'est plus croire en un fait accompli (ibid.).

Cette position est capitale: là où, dans nombre de paradigmes scientifiques dominants, les scientifiques sont perçus comme des «intermédiaires plus ou moins fidèles », définis par leurs «inputs » et «outputs », B. Latour leur fait jouer le rôle de «médiateurs, c'està-dire des acteurs dotés de la capacité de traduire ce qu'ils transportent, de le redéfinir, de le redéployer, de le trahir aussi " (1991, p. 111). Le scientifique, dans cette perspective, ne peut plus être mis en boîte noire pour être oublié et ignoré tout à fait. La traduction désigne ici le travail de référence par lequel des entités, des inscriptions, des énoncés hétérogènes, sont mis en relation, travail dont le succès n'est jamais assuré, et qui détient ses propres critères de validité :

L'explication de la force d'un énoncé, c'est-à-dire de sa capacité à convaincre et à s'imposer dans une controverse, va de pair avec l'explication de sa signification. Celle-ci tient aux chaînes de traduction et aux références qu'elles construisent, celle-là est mesurée par la robustesse de ces chaînes et plus particulièrement par la morphologie des réseaux qu'elles composent. Un énoncé isolé n'a pas plus de force qu'il n'a de sens (Callon, 1995, p. 245).

L'évacuation du critère de réfutabilité, qui centralise tant de débats sur la caractérisation de la scientificité, ne conduit pas pour autant à un relativisme selon lequel tous les critères se valent, et qui postule que nous ne pouvons jamais nous approcher fidèlement de la réalité extérieure (accusation fallacieuse régulièrement portée à $\mathrm{B}$. Latour, qui défend quant à lui un plus grand réalisme). L'exigence de rigueur se porte, dans le modèle traductif, sur la capacité des énoncés à résister à d'éventuelles « contretraductions ». L'introduction de ce critère d'irréversibilité parmi ceux qui fondent le régime de scientificité, en contrepoint de celui de réfutabilité, a ceci d'intéressant qu'il désavoue l'hypothèse d' " une place pour chaque chose et chaque chose à sa place », pour des entités que les mots sont chargés de saisir, de maîtriser, de fixer dans une adéquation avec le monde qu'ils représentent. Là où la rationalité, adossée à la réfutabilité, s'intéresse à l'énoncé final, qui ne constitue qu'un point d'aboutissement provisoire d'une chaîne de traduction, d'une série de représentations successives et enchâssées, le modèle traductif insiste quant à lui sur la stabilisation des chaînes de traduction :

Un réseau devient irréversible à proportion que les traductions qu'il déploie augmentent leur degré de consolidation et de robustesse, rendant les traductions suivantes prévisibles et inévitables (ibid., p. 248).

Dans cette perspective, l'entité approchée — pour ce qui nous retient ici, la vie psychique dans toute sa pluralité et sa complexité - dont nous nous efforçons de recueillir les traces pour les transformer en un énoncé qui décrit ses propriétés, n'est pas considérée «comme la cause de l'énoncé » mais est analysée
« comme ce qui circule dans la chaîne de traduction » (ibid., p. 241). Dès lors, l'exposé méthodologique consiste à soutenir l'effort de description non plus du côté des résultats attendus à partir d'énoncés aptes à les prédire, mais sur les montages, non exhaustifs, par lesquels les énoncés sont produits soulignant la part processuelle de la démarche. De là s'impose une exigence de description détaillée des dispositifs qui les produisent, non pour être reproduits, mais pour être discutés : l'exposé méthodologique, en ce sens, ne vise pas à mettre fin à la discussion sur l'opportunité de ces montages, mais à préparer au contraire les conditions d'une telle discussion, en s'efforçant de mettre à disposition, de rendre accessibles et de partager, au plus près de l'expérience, les tâches concrètes empiriques du chercheur et les bases à partir desquelles est mené le travail de traduction. C'est à cette condition que ce travail sera susceptible d'ê tre repris (et non reproduit) par d'autres chercheurs qui pourront, à la lumière de leurs propres enquêtes, proposer de nouvelles traductions. Ce ne sont donc pas les résultats en tant que tels qui sont généralisables, mais la possibilité d'utiliser les produits de la recherche pour l'étendre à d'autres questions et contextes de recherche. Cette approche, ainsi, est loin de réduire ou d'écarter le dissensus d'évaluation, sous prétexte d'incommensurabilité de praxis hétérogènes, et de la vanité de leur ordonnancement au regard de la question qui leur est adressée.

Ces propositions nous éloignent donc d'un modèle linéaire de la recherche, dans lequel une recension exhaustive de la littérature et la récapitulation des résultats de recherches antérieures, pris comme modèles à vérifier, déboucheraient sur des hypothèses probabilistes de recherche, suivant la démarche suivante (Fig. 1).

Dans notre perspective, les hypothèses princeps constituent moins des hypothèses de travail, formelles, stabilisées, que des hypothèses en travail, processuelles, transformant l'objet d'étude et son abord au fur et à mesure de la recherche, au fur et à mesure des épreuves proposées par le chercheur au sein du dispositifde recherche (entretiens invitant les sujets à penser leur situation par exemple). Le travail de problématisation conceptuelle ne se nourrit pas seulement des allers-retours entre compréhension et expérience, mais naît de ce mouvement même, incessant, de reprise, située toujours entre deux moments d'une praxis (exemple ici à partir du dispositif de recherche d'une étude doctorale portant sur le vécu de la dépendance dans le grand vieillissement) (Fig. 2).

Ce mouvement n'exclut pas pour autant complètement le modèle linéaire, qui peut rester mobilisable localement, ponctuellement, à l'intérieur des cycles successifs. Dans le modèle tourbillonnaire cependant, les phases de conceptualisation, d'expérimentation et de transformation ne sont pas strictement séparées, mais au contraire bigarrées de multiples assemblages. Ainsi, l'élaboration progressive du cadre conceptuel impacte notre compréhension de ce que l'expérience de recherche clinique nous donne à voir au fur et à mesure, tant elle influence la manière dont nous écoutons les sujets que nous rencontrons successivement et dont nous saisissons les processus psychiques susceptibles de se déployer dans l'espace des rencontres de recherche.

Sans établir d'équivalence, soulignons cependant que la modélisation spatiale du processus de recherche ici mise en avant fait écho au modèle de la spirale proposé par J. Laplanche comme dimension essentielle de son modèle traductif: «La spirale est une courbe pleine qui décrit des révolutions concentriques, à partir

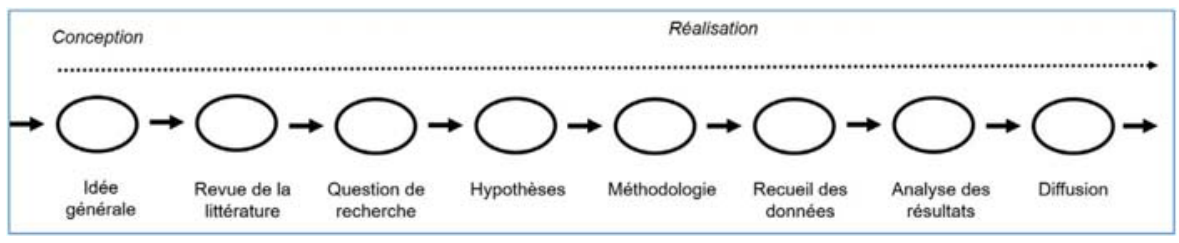

Fig. 1. (extrait de Racin, 2017). Modèle linéaire : enchaînement irréversible d'étapes successives (adapté de Akrich, Callon, \& Latour, 1988, p. 21). 


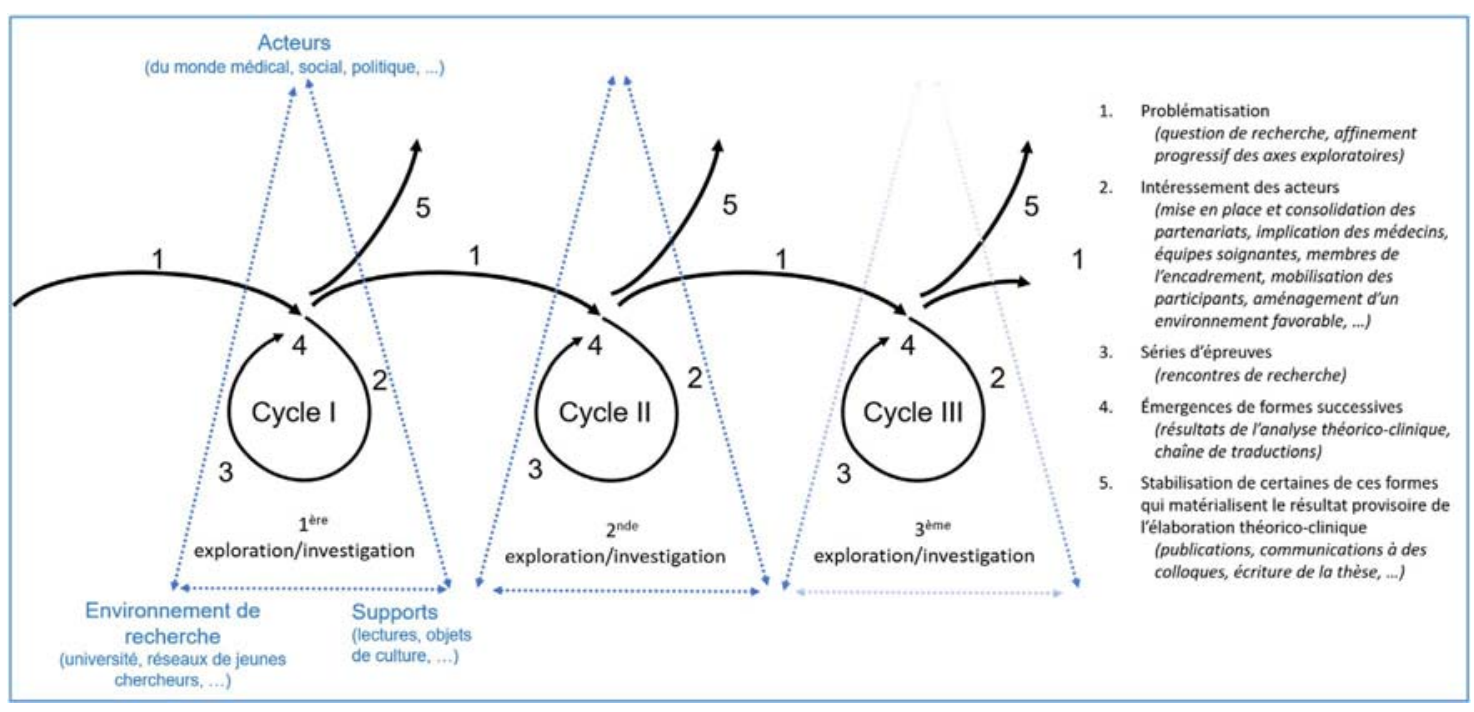

Fig. 2. (extrait de Racin, 2017). Modèle processuel : transformations successives du projet initial (adapté de Akrich, Callon, \& Latour, 1988, p. 21).

d'un point nommé pôle, révolutions de plus en plus élargies » (1980, p. 6). Ce mouvement concentrique, tout en s'éloignant de plus en plus du point de départ pour ouvrir à d'autres significations et d'autres compréhensions des processus engagés dans la recherche, offre une autre mise en forme de la dimension processuelle de la recherche. Avant de constituer un axe théorique central des travaux de J. Laplanche avec son modèle traductif, la traduction occupe une place importante dès les débuts de la psychanalyse $^{4}$ avec Freud qui l'inscrit comme moteur du refoulement dans la lettre à Fliess 52/112, datée du 6 décembre 1896 : «le refusement de la traduction, c'est cela que cliniquement on nomme le "refoulement" "(1887-1904 p. 265). Comme y insiste J.-B. Pontalis, «qu'on le veuille ou non, le traduire est toujours en jeu dans l'interprétation et même dans l'écoute. On pourrait aussi avancer que toute nouvelle théorisation de l'analyse naît de l'insatisfaction devant les traductions précédentes »(1977, p. 192). Ainsi, la traduction a toute sa place dans des recherches se référant à la psychanalyse. La traduction est au cœur de la vie psychique pour Freud, dans le rêve d'abord, mais aussi dans la psychopathologie de la vie quotidienne et ses actes manqués, tout comme on la retrouve dans les symptômes. "Il faut considérer Freud comme l'un des plus importants théoriciens de la traduction car il a donné à ce concept une envergure et une profondeur sans précédent dans l'histoire. À travers l'utilisation du mot allemand Übersetzung dans sa double signification de traduction et de transposition » (Mahony, 1987, traduit par Z. Andrejew, 2018). Elle est à ce titre un travail psychique porteur de transformation voire de symbolisation. J. Laplanche, traducteur de Freud, propose une clinique de la traduction, mais aussi un modèle traductif comme support méthodologique d'accès à l'œuvre de Freud et à l'inconscient, notamment dans l'alternance traduction/détraduction/ retraduction: « la traduction comme pratique avec les œuvres complètes de Freud [...]. La traduction comme modèle analytique inéluctable, lorsqu'il s'agit de la constitution de l'appareil d'â me, et du refoulement mais aussi de l'interprétation, de la sublimation etc. .. » (1992, p. 309). La proposition de J. Laplanche ouvre à prendre en compte la traduction comme méthode d'appréhension de la vie psychique en tant que processus de transformation et de prise en compte du fonctionnement psychique dans son altérité

\footnotetext{
${ }^{4}$ On peut se référer à la thèse de doctorat de psychologie de Z. Andrejew : «La question de la traduction dans la théorie et la clinique psychanalytiques », soutenue le 19 novembre 2018 à l'université Paris Descartes.
}

constitutive, car infiltré par un sexuel infantile dont les formes d'expression sont autant d'idiomes en attente de traduction.

Laboratoire central et recherches cliniques : pour une méthode de recherche hypothétique et processuelle

Toutes les nuits entre onze et deux heures, je n'ai fait que fantasmer, transposer et deviner (1887-1904, Lettre à Wilhelm Fliess du 25 mai 1895).

La reprise du titre de l'ouvrage de J.-B. Pontalis nous permet de poursuivre notre réflexion sur les enjeux spécifiques et les formes prises par des recherches cliniques se référant à la psychanalyse. Comment formaliser ces recherches, comment y maintenir une dimension scientifique? L'idée d'un laboratoire central comme métaphore de l'appareil psychique, mais aussi comme outil à la disposition du chercheur, semble une piste féconde, à la condition de réaffirmer, à la suite de D. Scarfone, qu' « il n'y pas entre deux humains de pure observation qui ne serait pas elle-même perçue par l'observé comme signifiant une intention de l'observateur, et se faisant en retour elle-même objet d'observation " (op. cit., p. 111). La prise en compte à la fois de la dimension intersubjective, de ses effets sur la recherche et de la transformation de la situation observée du fait même de son observation, se trouve au cœur des méthodes cliniques référées à la psychanalyse, tout comme le sont les modes d'accès à de nouvelles formes de pensées et de connaissance ouverts par l'interprétation, le devinement et la rêverie - au sens décrit par Bion à la fois comme capacité de contenance et d'accueil des contenus psychiques les moins différenciés et de leur possible transformation: "la rêverie est un facteur de la fonction alpha de la mère »(1962, p. 54).

Pour autant, il ne s'agit pas de dupliquer la méthode de la cure analytique sur une recherche, mais plutôt de mettre à l'épreuve processus de la cure et processus de la recherche. Pour R. Roussillon, « au postulat isomorphique des pragmaticiens, la psychanalyse oppose le postulat homomorphique. À l'identité de perception, la démarche freudienne substitue le travail de construction d'une identité de pensée " (2001a, p. 50). En algèbre générale, un morphisme (ou homomorphisme) est une application entre deux structures algébriques de même espèce 
- c'est-à-dire des ensembles munis de lois de composition interne ou externe (par exemple deux groupes ou deux espaces vectoriels) - qui respecte certaines propriétés en passant d'une structure à l'autre. Plus généralement, la notion de morphisme est l'un des concepts de base en théorie des catégories; ce n'est alors pas forcément une application, mais une "flèche » reliant deux «objets» ou «structures» qui ne sont pas forcément des ensembles.

Notre démarche s'inscrit dans cette affirmation d'un homomorphisme entre processus psychanalytique et processus de recherche : les deux structures sont différentes mais gardent des propriétés et des formes semblables. Parmi celles-ci, nous en développerons deux : d'abord la place du processus et de la processualité dans la recherche, ensuite le nécessaire maintien d'un écart entre théorie et clinique et à l'intérieur de la recherche, par exemple à l'appui de la formulation initiale d'hypothèses.

Processus, processualité et « procéder » en recherche clinique

Comme l'affirme G. Lanteri-Laura (1958), le processus a été, dans 1'histoire des idées, une solution proposée par la psychopathologie aux impasses des causalités prônées par les modèles médicaux. L'intérêt en clinique psychanalytique pour les processus s'inscrit notamment dans les travaux de R. Roussillon (2001b) sur l'approche métapsychologique des processus, sur l'étude du processus analytique et de ses avatars, mais aussi sur ce qu'A. Brun nomme la $4^{\mathrm{e}}$ vague d'évaluation des psychothérapies. Celle-ci place les processus au cœur de l'évaluation en examinant leurs transformations au sein du fonctionnement psychique - par ou dans un dispositif thérapeutique, par ou dans une situation clinique - et plus uniquement sur la modification des symptômes ou des comportements. L'équipe de Lyon 2, autour de R. Roussillon et A. Brun, a développé depuis plusieurs années des recherches centrées sur les processus, recherches intriquées à leurs travaux sur les processus de symbolisation. Ainsi, ils promeuvent des recherches sur et par les processus et se situent dans une épistémologie de la recherche centrée sur 1'approche clinique, dans une spécificité par rapport aux recherches issues du courant médical de l'évaluation: «on part au contraire des pratiques pour dégager la preuve, "la preuve est fondée sur les pratiques", et non pas l'inverse »(Brun, 2016, p. 4). L'évaluation s'appuie, pour R. Roussillon (2016), sur une mise en tension et sur une dialectisation entre analyse de l'associativité et analyse des processus en jeu.

Notre proposition met en avant l'importance de la prise en compte des processus tels qu'ils se déploient dans la vie psychique et dans le modèle spécifique de la cure type et de ses variations. Pour Freud, la psychanalyse est un procédé et permet un " procéder "d'investigation spécifique des processus psychiques inconscients. Ce procédé nous semble être, par homomorphie, un modèle de recherche processuel qui postule d'emblée la complexité et l'appréhension asymptotique de l'inconscient, ainsi que le maintien d'un écart théorico-clinique ou théorico-pratique. Comme nous 1'avons déjà souligné, une approche épistémologique des processus les place comme solution déplaçant les butées théoriques et les frontières des méthodes habituellement utilisées dans la compréhension des phénomènes pathologiques. Les processus offrent une voie nouvelle d'appréhension de ces phénomènes. Une méthodologie hypothétique et processuelle s'intègre dans cette proposition d'ouverture sur la complexité psychique et sur la place centrale accordée aux processus et à la processualité dans la psyché, ainsi que dans les dispositifs thérapeutiques s'offrant comme des déclinaisons de la cure type. Promouvoir une méthodologie processuelle ouvre sur une dimension dynamique, sur un "en cours de construction "sur un inachevé, une ouverture à poursuivre et à continuer à chercher. « Parler de processus signifie ouvrir le discours vers des événements qui se développent selon des directions possibles [...] d'une ouverture vers l'inconnu et l'indéterminé, vers ce qui n'a pas encore été pensé, vers un possible imprévisible " (Petrella, 2004 , p. 1559). Nous pouvons reprendre cette proposition de F. Petrella comme ligne directrice d'une méthode processuelle ouvrant sur une investigation de la vie psychique, des enjeux mobilisés à certains âges de la vie, des problématiques suscitées face à certains événements de vie, mais aussi sur une investigation des mouvements psychiques complexes susceptibles de se déployer au sein de dispositifs thérapeutiques centrés sur une transformation de la vie psychique (psychothérapie, cure analytique, dispositifs groupaux à médiation...).

Il n'existe pas à proprement parler, dans l'œuvre de Freud, de théorie ou de conceptualisation du ou des processus. Il y fait cependant référence à de multiples reprises, selon deux grands axes : celui des processus psychiques en tant que tels - à l'instar de l'opposition entre processus primaires et processus secondaires et celui du processus analytique. « Dès le début de l'œuvre de Freud, et ensuite dans la psychanalyse postfreudienne, nous rencontrons sans cesse des références, presque toujours explicites, à des processus, tant pour indiquer des orientations fonctionnelles générales de la psyché [...] que pour qualifier des phénomènes plus partiels, qui ont besoin d'une élaboration métapsychologique car il s'agit de processus inconscients. [...] Mais la psychanalyse elle-même, dans son ensemble, est un processus »(ibid., p. 1562). Il y aurait une cohérence épistémologique à penser la recherche en psychanalyse comme une recherche processuelle, c'est-à-dire comme contenant - au sein de son dispositif et de sa méthodologie - une part processuelle, mais aussi comme attracteur ou révélateur des processus psychiques favorisant ainsi leur exploration.

À l'instar de la cure, la recherche est porteuse d'un courant processuel par nature. Dès lors, il serait simplificateur de postuler à une dynamique passive du chercheur porté par ce courant, tout comme l'analyste et l'analyse chemineraient le long du processus analytique. La méthode de recherche, comme la méthode analytique, relèvent d'une construction, et même plus encore d'une co-construction du chercheur et des sujets de sa recherche, de l'analyste et de l'analysant. D. Scarfone, en reprenant les propositions freudiennes de l'Esquisse (Freud, 1895) à propos du traitement de l'excitation, insiste sur le frayage en cours dans le processus analytique, sur la part active qu'elle implique et sur les résistances qu'elle pourra y rencontrer. Il ne s'agit donc pas de déambuler dans la recherche, porté par une associativité totalisante, mais plutôt de tracer son chemin, ici la méthodologie, puisque pour D. Scarfone : "s'il faut parler de processus analytique, celui-ci n'est pas tant un chemin que le traçage et le forage de nombreux chemins » $(2004 b$, p. 1630). La part processuelle de la recherche s'étaye sur la théorie qui participe très largement au frayage d'une voie à travers la multiplicité des références théoriques. La part subjective du chercheur y est alors fortement engagée, notamment dans le choix qu'il fait de telle ou telle approche, de tel ou tel concept. S'y trouvent également mêlées les propres théories du chercheur qui, comme les théories sexuelles infantiles, infiltrent les rencontres théoriques ultérieures. Le traçage proposé par D. Scarfone pour appréhender la dynamique processuelle se déploie aussi sur la méthodologie de la recherche dont les hypothèses peuvent se concevoir comme les supports de progression dans la jungle de l'inconscient. Elles constituent la première direction prise par le chercheur dans le prolongement des concepts théoriques mis en avant. Elles énoncent les choix à l'origine du processus de recherche: première mise en forme des propositions ou des élaborations du chercheur face à telle problématique ou telle situation clinique. L'approche processuelle contient ainsi une part dynamique, déterminée par la "force » engagée dans la recherche, et une part de mise en sens portée par la 
pensée du chercheur, telle qu'elle apparaît dans la forme prise par la méthodologie et les hypothèses. Il existe une dialectisation à l'intérieur même de la notion de processualité : celle-ci peut être appréhendée en tant que force, vecteur, chemin, direction, aller et retour entre surface et profondeur; elle peut également l'être en tant que forme, sens, transformations et déformations induites par la contrainte de la méthode psychanalytique. Cette dernière est porteuse d'un paradoxe qui se déplace jusque dans la recherche, d'un côté dans l'appréhension des phénomènes étudiés, leur représentation et présentation par le chercheur, et de l'autre côté dans l'illusion de pouvoir les circonscrire avec le risque de les figer et de les réduire, quitte à perdre leur spécificité.

De nombreux travaux ont porté sur le dispositif analytique en y différenciant le cadre et le processus, respectivement part fixe et part variable de la cure. Progressivement, l'intérêt s'est déplacé vers la notion de processus analytique, mise en tension avec celles de contre-processus et d'anti-processus. Dans une première approche de la cure des névrosés, le processus permet, par la levée du refoulement et par l'installation de la névrose de transfert - en restant au plus près du modèle historique proposé par Freud -, d'accéder à l'inconscient en rendant conscients des contenus psychiques inconscients, notamment par la transformation des processus primaires en processus secondaires. La cure est aussi un "procédé », une méthodologie originale d'investigation de la vie psychique. Cette part invariante repose sur la référence épistémologique à la psychanalyse. "Procéder en psychanalyse ", pour F. Petrella, consiste à porter une attention égale, au-delà du scopique et du manifeste de l'écoute, aux dimensions consciente et inconsciente, subjective et intersubjective, transférentielle et contre-transférentielle. Il s'agit de procéder par interprétation et construction au niveau manifeste, et par intuition et devinement ${ }^{5}$ au niveau latent. Une recherche hypothétique et processuelle contient cette même distinction et mise en tension entre sa part fixe - le dispositif méthodologique de recherche - et sa part variable - le processus.

Le choix d'un référentiel analytique dans la recherche clinique ne peut se satisfaire pleinement d'un recours aux méthodes hypothético-déductive et ancrée. La proposition de la constitution d'une méthode processuelle sort de l'opposition classique entre objectivité et subjectivité de la recherche. Elle se base sur une mise en avant de la compréhension des phénomènes psychiques et de leur complexité qu'une recherche centrée sur une causalité directe risquerait de réduire. Il s'agit ici de prendre en compte plusieurs axes d'observation, d'investigation et d'analyse : le récit du sujet en tant que premier indicateur des processus psychiques mobilisés, l'associativité qui s'y déploie et toutes formes de langage utilisées par le sujet. D'autres supports d'exploration du fonctionnement psychique - grilles d'entretien, échelles, tests projectifs... peuvent également être utilisés comme voies d'accès complémentaires aux processus psychiques. La dimension processuelle de la recherche ouvre aussi à une temporalité plus à l'écoute de la vie psychique, où règnent la discontinuité et l'après-coup. Ils infiltreront le processus de recherche à travers les temps multiples: d'élaboration des hypothèses au cours de la réflexion méthodologique, de recueil et de traitement des données, d'analyse et de discussion des résultats, de rédaction et de présentation de la recherche. "L'espace processuel qu'il faut construire et traverser est étendu et virtuel, temporel et atemporel, répétitif, régressif et potentiellement évolutif et propulsif » (Petrella, op. cit., p. 1569). Il est support de potentialité mais aussi de coup d'arrêt, de répétition voire d'impasses.

5 «Devinement » se situe dans la proposition freudienne du « deviner», «erraten », reprise par M. Gribinski (2004).

\section{Hypothèses et écart théorico-clinique}

La cure, dans la proposition freudienne, au-delà d'être un outil thérapeutique au service de la transformation et du changement de la vie psychique, en est une modalité privilégiée d'observation - de ses bigarrures et de ses achoppements. Il est intéressant de reprendre la distinction mise en avant par A. Green entre processus psychique et processus analytique, ce dernier renvoyant à un "mode d'organisation dynamique des phénomènes du psychisme, [...] et qui est supposé qualifier la progression de la cure » (2004, p. 1871). Il s'agira donc, dans notre construction méthodologique, de pouvoir prendre en compte à la fois l'organisation dynamique des sujets observés, d'une part, la progression de la recherche et du chercheur confronté à cette dynamique, d'autre part. Ce positionnement rend compte de l'écart théorico-clinique inhérent au référentiel analytique : écart entre le chercheur clinicien et le chercheur aux prises avec l'analyse de ses résultats, mais écart aussi entre la formulation des hypothèses avant le début de la recherche et au moment de la rédaction. Il ne s'agit pas de réduire cet écart mais de le souligner et de le prendre en compte en raison de sa fécondité sur les résultats de la recherche. En cela, cette méthodologie se différencie d'une méthodologie inductive, telle qu'elle se déploie dans la théorisation ancrée, où la confrontation au matériel permet la constitution de catégories qui se transforment au fur et à mesure des nouveautés rencontrées, ce qui abrase tout écart et fait courir le risque d'une circularité : ce qui est démontré est ce qui a été observé et ce qui était attendu. Au contraire, il nous semble plus dynamique, dans notre proposition méthodologique, de faire un retour aux hypothèses après avoir fait un détour par les données recueillies, dans le prolongement de ce que A. Green propose de l'analyse en tant que retour sur soi via un détour par 1'autre. Le chercheur revient à ses hypothèses initiales, transformées en résultats qui peuvent générer de nouvelles hypothèses ou propositions de recherche, suivant une séquence " liaison/déliaison/reliaison " classique dans le processus analytique: «le processus est processus par sa visée de procéder par l'exploration pluridirectionnelle pour la connaissance de l'objet et sa réflexion sur le sujet qui met en œuvre cette exploration ", comme le propose A. Green (ibid., p. 1874).

On peut s'interroger sur la volonté de garder, dans cette proposition méthodologique, le recours à des hypothèses alors qu'elles sont décriées en raison notamment de leur forte intrication à une représentation-but du chercheur : leur validation. Au même titre que la règle fondamentale postule la suspension des attentes de l'analyste, les hypothèses gardent leur pertinence en tant que mise en forme par le chercheur des processus qu'il souhaite étudier. Ainsi, comme l'affirme J.-L. Donnet, « la suspension requise de la représentation de but consciente doit permettre, à travers l'association libre, la manifestation de processus psychiques inconscients et de leur jeu de forces conflictuelles " (2004, p. 1897). Toujours en paraphrasant une autre de ses propositions, il semble que l'enjeu de la recherche est de construire un dispositif promoteur des processus psychiques et de leur interprétation. La mise en place d'hypothèses organise et condense ces processus autour d'un type de questionnement: le double implicite d'une non-validité potentielle et d'une non-exhaustivité de l'hypothèse maintient l'écart théorico-pratique indispensable à toute recherche. La méthode, au plus près de la méthode analytique conçue par Freud, postule « une disjonction fonctionnelle entre sa mise en œuvre rigoureuse et les résultats, conçus comme les effets indirects, médiats, des transformations psychiques accomplies » (ibid., p. 1898) pendant la recherche. Mais comme pour l'analyse, la dimension processuelle n'est qu'une construction : le leurre qui viserait à saisir l'ensemble de la psyché d'un sujet cède la place à la tension asymptotique vers ce saisissement. Il faut aussi s'attendre 
à — voire même permettre — l'émergence de résistances induites par le dispositif de recherche, la survenue d'une part antiprocessuelle et contre-processuelle, du côté des sujets comme du côté du chercheur. Le remodelage des hypothèses s'inscrit dans cette logique anti-processuelle et peut rendre compte de difficultés liées à la confrontation à cet écart, les pré-attentes du chercheur se dévoilant dans l'après-coup à travers cet impératif de « réajustement ».

Le maintien des hypothèses dans le dispositif de recherche nous semble un élément central de celui-ci, en ce qu'elles permettent une décondensation de la complexité inhérente à toute matière psychique. Là où, dans le modèle hypothético-déductif, les hypothèses relèvent de construits du chercheur qui interrogent a priori la réalité supposée objective d'un phénomène en appelant une réponse probabiliste qui ne rend pas compte de la structure ontologique intime du processus, nous proposons d'utiliser les hypothèses comme axes d'analyse. Dans cette perspective, le travail d'hypothèse ou l'hypothèse en travail permet une approche structurale et dynamique en raison de la fragmentation qu'elle favorise, de ce passage détail par détail comme modalité $\mathrm{d}$ 'approche $\mathrm{du}$ fonctionnement psychique dans son ensemble. Certes, la construction d'hypothèse est aussi porteuse d'une menace d'objectivation au sein de la méthode analytique, de standardisation de la démarche et de réification des concepts au profit d'une simplification réductrice. L'approche processuelle dans laquelle sont prises les hypothèses tente de réduire ce risque, la stabilisation des hypothèses tout au long de la recherche et l'incontournable mise au travail par le chercheur de l'écart qu'elles entraînent dans l'analyse des résultats en étant quelques-uns des pivots. La nécessaire reproductibilité de la mise au travail des hypothèses pour chaque sujet et tous les sujets de la recherche participe à faire de la méthode hypothétique et processuelle une méthodologie générant une connaissance de/ sur la psyché. Comme le souligne J. Laplanche à propos de l'exigence freudienne de décomposition recomposition (1993, p. 9), la reproductibilité est présente au niveau méthodologique : si les cas sont singuliers, la méthode d'approche est la même.

La variété des modalités d'accès à la connaissance conduit à délimiter habituellement deux grandes catégories de méthodologie de recherche : déductive et inductive ${ }^{6}$. Cette dichotomie poursuit notamment l'opposition épistémologique entre connaissance objective et connaissance subjective. Non soluble dans ces deux modalités sans pour autant pouvoir en être dissociées, l'approche freudienne explore et formalise la voie de l'interprétation (Lanteri-Laura, op. cit.) voire de devinement (Gribinski, op. cit.). Notre proposition d'une méthodologie hypothétique et processuelle reprend cette proposition de recours à l'interprétation des résultats. Le mécanisme d'interprétation se conçoit en référence à l'action de l'analyste et au-delà, à la situation anthropologique originaire. Pour J. Laplanche, les signifiants énigmatiques présents dès les débuts de la vie placent l'être humain en position d'interprète des expériences qu'il rencontre. En ce sens, «c'est une herméneutique fondatrice que nous postulons : la situation de quelqu'un à interpréter, à donner sens à "ce qui lui arrive" [...]. Qu'est-ce qui m'arrive ? Commente le maîtriser en me l'appropriant par une "traduction"” "(1997, p. 1187). L'interprétation est à la fois traduction et construction; elle ouvre sur la potentialité de l'approche processuelle et sur son opposition à une vision

\footnotetext{
${ }^{6}$ Pour tenter de «réconcilier » ces deux approches, un compromis apparaît de nos jours dans 1'application d'un raisonnement dit abductif, dont la référence s'est développée à partir des travaux de Charles Sanders Peirce (1931-1935), regroupés et publiés post-mortem. Ce raisonnement s'inscrit dans une "logique de la découverte ", au sein de laquelle déduction, induction et abduction ne s'opposent pas, mais se combinent en pratique, selon une grande disparité de compréhensions et d'utilisations cependant.
}

totalisante et conclusive induite par une validation/non-validation des hypothèses de recherche.

\section{L'écriture de la recherche : entre processus et écart}

Le dernier temps du travail de recherche passe par l'écriture. Ce temps de rédaction redouble les effets de processualité et d'aprèscoup : "si l'écriture représente un stade conclusif, tant dans le parcours de formation que dans l'activité de recherche, bien loin d'être une transcription syntaxiquement organisée d'une séquence d'événements et de pensées, le processus à travers lequel nous arrivons à produire l'écriture est tel qu'il parvient à transformer le processus même qui l'a engendré, qu'il s'agisse de la processualité du travail analytique dans la rédaction des matériaux cliniques ou qu'il s'agisse de l'élaboration d'aspects théoriques " (Munari, 2004, p. 1773). Le temps d'écriture mobilise également la part processuelle de la recherche, tout comme les effets d'après-coup contenus dans l'écart théorico-pratique de cette méthodologie de recherche. Elle contraint le chercheur à un arrêt au terme de la temporalité de la recherche, mais elle ouvre aussi sur un temps de suspension propice à la reprise d'une position de chercheur, voire de nouvelles recherches.

C'est donc la périodisation de la réflexion du chercheur, bordée par la temporalité de sa recherche, qui délimite l'aboutissement provisoire de la trajectoire de recherche — par ailleurs définitivement condamnée à l'inachèvement (ce dont nous pouvons nous réjouir !) - et se stabilise sous la forme de propositions inaugurales. Ces dernières, rendues transmissibles et discutables par ce que J.-F. Chiantaretto appelle «l'exercice d'une discursivité critique ", ne relèvent pas d'un simple exposé, mais d'une écriture impliquée du clinicien-chercheur : «il s'agit d'aborder l'écriture en tant qu'elle constitue un lieu psychique externalisé, matérialisant un espace intersubjectif : indissociablement un espace pour penser ses pensées, doté d'une relative autonomie par rapport à la psyché, et la surface d'inscription d'une parole adressée " (2004, p. 174

175). Il ne s'agit pas de déterminer si ces propositions sont vraies ou fausses, mais si elles sont bien ou mal articulées. Insister sur l'articulation des propositions revient à insister, au-delà du repérage des régularités ou des similitudes dans les données d'observation, sur la recherche de mise en cohérence, à partir de laquelle l'« utilité » de ces propositions est validée dans la compréhension de bien des faits que leur absence, comme le souligne R. Perron (2008), ne permet pas de comprendre, leur récusation réduisant alors considérablement le champ phénoménal. Pour poursuivre cette idée avec D. Widlöcher, «le clinicien ne cherche pas à valider les concepts, il les utilise pour valider sa compréhension de ce qu'il observe. Tout juste montre-t-il qu'un concept lui est utile. En clinique, la validité d'un concept est testée en fonction de son utilité. La méthode clinique questionne l'expérience et utilise des concepts pour formuler une réponse, provisoire certes, mais utile en l'état de l'expérience commune » (2004, p. 16). En ce sens, le chercheur n'en a jamais véritablement fini avec la traduction. L'élaboration et l'exposition méthodologiques visent donc, d'une part, à inventer un cheminement à la fois rigoureux et capable d'intéresser la communauté scientifique; à attirer l'attention, d'autre part, sur les conditions dans lesquelles les énoncés sont produits. Cette élucidation, qui implique de s'extraire d'un vécu "impressionniste " pour indiquer où se situent les traductions, revêt un caractère obligatoire si nous voulons nous assurer nos «données ». Ce dernier point exige, dans le travail d'écriture, un effort d'analyse et d'exposition singulier concernant les caractéristiques du cadre externe et du cadre interne du chercheur qui permettent d'obtenir et d'assurer justement ces données. Cela suppose notamment, pour le chercheur, de prendre en compte les résistances externes et internes (subjectives, éthiques...) contribuant aux transformations qui affectent (au sens de produire un effet sur) le processus de recherche. 


\section{Perspectives}

L'emprunt d'éléments disparates à des paradigmes dominants, autorisé par la grande diversité de leurs lectures possibles, semble en apparence résoudre l'opposition tranchée entre réalisme et constructivisme, entre déduction et induction, entre subjectivisme et objectivisme, entre démarche qualitative et démarche quantitative, etc., au profit d'un dépassement de ces dichotomies dans l'établissement d'un pseudo-continuum qui ne fait que poursuivre les mêmes types de pensée et de rapport à la réalité. Ce positionnement, qui apparaît de fait très souvent pour dépasser les contraintes rencontrées au cours de l'opérationnalisation, conduit in fine à aménager différents paradigmes au sein d'un syncrétisme qui se pare des attraits de la fécondité quand, sur un mode défensif, il esquive la nécessité de rendre compte de la spécificité de la connaissance scientifique référée à la psychanalyse, des difficultés inhérentes à sa dynamique de production singulière. Ces tensions nous semblent importantes à prendre en considération dans le processus de construction du savoir auquel nous souhaitons que la recherche clinique référée à la psychanalyse contribue. C'est à cette démarche que nous souhaitons contribuer, à travers la modélisation d'une " méthode hypothétique et processuelle» qui rencontre dans cet article un premier moment de formalisation.

\section{Déclaration de liens d'intérêts}

Les auteurs déclarent ne pas avoir de liens d'intérêts.

\section{Références}

Akrich, M., Callon, M., \& Latour, B. (1988). À quoi tient le succès des innovations ? Deuxième épisode : L'art de choisir les bons porte-parole. Gérer et comprendre. Annales des mines, 12, 14-29.

Andrejew, Z. (2018). La question de la traduction dans la théorie et la clinique psychanalytiques (Thèse de doctorat de psychologie) Université Paris Descartes.

Ansermet, F., \& Magistretti, P. (2004). À chacun son cerveau. Plasticité neuronale et inconscient. Paris: Odile Jacob.

Atlan, H. (1979). Entre le cristal et la fumée. Essai sur l'organisation du vivant. Paris: Le Seuil, «Points».

Ayache, M., \& Dumez, H. (2011). Le codage dans la recherche qualitative: une nouvelle perspective? Le Libellio d'Aegis, 7(2), 33-46.

Bachelard, G. (1934). Le nouvel esprit scientifique (2013). Paris: PUF.

Bion, W. R. (1962). Aux sources de l'expérience (1979). Paris: PUF.

Bion, W. (1970). L'attention et l'interprétation (1974). Paris: Payot.

Borst, G., \& Cachia, A. (2018). Les méthodes en psychologie. Paris: Presses Universitaires de France.

Brun, A. (2016). Introduction. In A. Brun, R. Roussillon, \& P. Attigui (Eds.), Évaluation clinique des psychothérapies psychanalytiques. Paris: Dunod.

Callon, M. (1995). Quatre modèles pour décrire la dynamique de la science. In M. Akrich \& B. Latour (Eds.), Sociologie de la traduction. Textes fondateurs (pp. 201-251). Paris: Presses des Mines (2006)

Chiantaretto, J.-F. (2004). L'analyste chercheur impliqué. Recherches en psychanalyse, $1(1), 171-178$.

Cyssau, C. (1999). Fonctions théoriques du cas clinique: De la construction singulière à l'exemple sériel. In P. Fédida \& F. Villa (Eds.), Le cas en controverse (pp. 59-82). Paris: P.U.F.

De Luca, M. (2009). Approche psychopathologique des scarifications (Thèse de doctorat de psychologie). Université Paris Descartes.

Donnet, J.-L. (2004). Le postulat processuel et l'aventure de la méthode. Revue française de psychanalyse, 68(5), 1897-1900.

Freud, S. (1887-1904). Lettres à Wilhelm Fliess : 1887-1904 (2006). Paris: PUF.
Freud, S. (1895). Esquisse d'une psychologie scientifique. In La naissance de la psychanalyse (pp. 373-486). Paris: P.U.F (1956).

Freud, S. (1938). Abrégé de psychanalyse (1978). Paris: PUF.

Glaser, B., \& Strauss, A. (1967). The discovery of grounded theory. Chicago: Aldine Publishing.

Green, A. (2002). Pour introduire la pensée clinique. In A. Green (Ed.), La pensée clinique (pp. 9-34). Paris: Odile Jacob.

Green, A. (2004). L'idéalisation du processus et sa problématique véritable. Revue française de psychanalyse, 68(5), 1865-1875.

Gribinski, M. (2004). Deviner à peu près. Revue française de psychanalyse, 68(3), 897915.

Ivins, W. M. (1985). La rationalisation du regard. Culture technique, 14, 30-37.

Kalat, J. W. (1999). Introduction to psychology. Belmont, CA: Wadsworth.

Laplanche, J. (1980). Problématiques I : L'angoisse (1970-1973). Paris: PUF.

Laplanche, J. (1992). La révolution copernicienne inachevée. Paris: Aubier.

Laplanche, J. (1993). Le fourvoiement biologisant de la sexualité chez Freud (2006). Paris: PUF.

Laplanche, J. (1997). Buts du processus psychanalytique. Revue française de psychanalyse, 61(4), 1181-1194.

Latour, B. (1991). Nous n'avons jamais été modernes. Essai d'anthropologie symétrique. Paris: La Découverte.

Latour, B. (2009). Sur le culte moderne des dieux faitiches; Suivi de Iconoclash. Paris: La Découverte.

Latour, B. (2010). Cogitamus. Six lettres sur les humanités scientifiques. Paris: La Découverte.

Lanteri-Laura, G. (1958). Psychopathologie et processus. Évolution psychiatrique, 50(3), 589-610.

Mahony, P. (1987). Psychoanalysis and discourse London: Methuen and Tavistock.

Mucchielli, A. (2004). Compréhensive (approche). In A. Mucchielli (Ed.), Dictionnaire des méthodes qualitatives en sciences humaines et sociales (pp. 24-28). Paris: Armand Colin.

Munari, F. (2004). Processus d'écrire et processus psychanalytique dans l'écriture. Revue francaise de psychanalyse, 68(5), 1773-1779.

Paillé, P. (1994). L'analyse par théorisation ancrée. Cahiers de recherche sociologique, 23, $147-181$.

Passeron, J.-C., \& Revel, J. (2005). Penser par cas. Paris: EHESS

Pelaccia, T., \& Paillé, P. (2009). Les approches qualitatives: Une invitation à l'innovation et à la découverte dans le champ de la recherche en pédagogie des sciences de la santé. Pédagogie médicale, 10(4), 293-304.

Perron, R. (2008). La psychanalyse est-elle réfutable? Revue française de psychanalyse, 72(4), 1099-1111.

Perron, R. (2010). La raison psychanalytique: Pour une science du devenir psychique. Paris: Dunod.

Petrella, F. (2004). Procéder en psychanalyse. Images, modèles et mythes du processus. Revue française de psychanalyse, 68(5), 1555-1626.

Poenaru, L. (2018). Le raisonnement psychanalytique. Hybridation et coadaptation. In Analysis, 2, 171-176.

Pontalis, J.-B. (1977). Entre le rêve et la douleur. Paris: Gallimard.

Pontalis, J.-B. (2012). Le laboratoire central. Paris: Éd de l’Olivier.

Popper, K. R. (1934). La Logique de la découverte scientifique (trad. N. Thyssen-Rutten et P. Devaux : 1973). Paris: Payot.

Popper, K. R. (1972). La connaissance objective (1998). Paris: Flammarion.

Quine, W. V. O. (1977). Le mot et la chose. Paris: Flammarion.

Racin, C. (2017). De l'hospitalisation à l'institutionnalisation des soins de longue durée dans le grand vieillissement: Étude clinique, psychopathologique et projective du «travail de dépendance » (Thèse de doctorat de psychologie). Université Paris Descartes - Sorbonne Paris Cité.

Roussillon, R. (2001a). Paradoxes et situations limites de la psychanalyse. Paris: PUF.

Roussillon, R. (2001b). Le plaisir et la répétition : Théorie du processus psychique. Paris: Dunod.

Roussillon, R. (2016). L'évaluation clinique de la subjectivité. In A. Brun, R. Roussillon, \& P. Attigui (Eds.), Évaluation clinique des psychothérapies psychanalytiques (pp. 3759). Paris: Dunod

Scarfone, D. (2004a). À quoi œuvre l'analyse? Libres cahiers pour la psychanalyse, 9(1), $109-123$.

Scarfone, D. (2004b). Processus et procédé psychanalytiques: Vertus et limites de l'analogie. Revue française de psychanalyse, 68(5), 1627-1635.

Thurin, J.-M. (1996). L'observation clinique formalisée dans la recherche en thérapie psychanalytique. $2^{\mathrm{e}}$ Colloque Interface INSERM/FFP - 15 mars 1996

Widlöcher, D. (1990). Le cas, au singulier. Nouvelle Revue de Psychanalyse, 42, 285-302.

Widlöcher, D. (2004). Pour une étude critique des concepts. Recherches en psychanalyse, $1(1), 15-19$. 